\title{
Hiv TESTING IN METHADONE PATIENTS
}

ntravenous drug users (IVDUs) with human

immunodeficiency virus (HIV) infection are an important potential source of HIV infection through needle sharing and/or sexual contact. There is however a lack of data about HIV prevalence and incidence data for IVDUs in NSW.

The Epidemiology and Health Services Evaluation Branch, with the NSW Methadone Program, recently explored the feasibility of improving data on the HIV status of IVDUs by collecting patient HIV testing data from methadone prescribers in NSW.

The NSW Methadone Program is managed by the Directorate of the Drug Offensive (DODO) in conjunction with the Pharmaceutical Services Section of the NSW Health Department, Area Health Services and Health Regions. The Health Department is responsible for granting approval to doctors to prescribe methadone. Each prescriber's approval is reviewed every six months.

Patients receive treatment at public clinics, from approved private medical practitioners or in the prison methadone program. There are similar numbers of public and private patients in the program. Treatment is available in the Sydney metropolitan area, at several regional clinics and from prescribers at rural centres. The patients represent a sizeable (more than 4000 patients ${ }^{1}$ ) and accessible group of intravenous drug users and former users. While they are not necessarily representative of IVDUs in general, they may comprise up to one-third of the estimated 12,000 IVDUs in NSW 2 .

\section{HIV-RELATED POLICIES}

The program has documented policies on HIV testing among patients. These include: counselling, confirmatory testing of those with a positive HIV test and, when patients are being assessed for entry to the program, HIV-related risk assessment and HIV screening when requested by the patient. There is no policy on regular follow-up screening.

\section{EXISTING DATA ON HIV STATUS}

\section{Entry and exit forms}

Prescribers are required to submit to the Health Department a detailed entry assessment form and a termination form for each patient. They also update selected patient details, such as dosage, every six months for renewal of authority to prescribe methadone. These data are collated by the Department. Neither the termination form nor the six-monthly prescription update includes information on HIV status.

Although the entry form has a three-part question on HIV testing and status, the data it yields are inadequate for surveillance purposes. About half the prescribers do not complete the questions (completion of all items is not enforced) and the information is based on patient self-report so its reliability is uncertain. A 'Review of Methadone Treatment' form which is also due every six months includes questions on HIV status but compliance by doctors in completing this form is poor.

\section{HIV testing practices}

There is a variety of screening practices, which range from arranging HIV tests at the methadone clinic for virtually all patients to always referring patients elsewhere (such as clinies specialising in AIDS/HIV) for HIV-test-related action. Neither the Health Department nor DODO has collected systematic data on individual prescribers' or clinics' HIV screening practices.

\section{StUdy OBJeCtives}

Our chief objective was to develop and trial a new procedure for directly obtaining accurate, timely HIV surveillance data from methadone prescribers. The main data of interest were:
- HIV sero-conversion prevalence rates among methadone patients; and,
HIV sero-conversion incidence rates among methadone patients.

The data would also provide:

\begin{tabular}{l|l} 
Baseline data on HIV testing status (when and \\
where previously tested) for new patients; \\
Information on HIV testing practices of \\
methadone prescribers; and, \\
Opportunities to explore relationships between \\
HIV status and selected patient characteristics.
\end{tabular}

\section{METHODS}

Ten private prescribers and three public clinics agreed to complete four items of information on patients who had had an HIV test. Prescribers were selected for this study because they were likely to be interested and/or cooperative. Participation was voluntary and of those approached, only one refused to take part. Some prescribers offered to take part without being approached. We discussed the study with the individual prescribers to confirm their co-operation and commitment.

We supplied specially designed data collection forms on which the prescribers recorded, for each patient who had an HIV test, the month and year of the most recent test, where the test took place, the result and whether the result was verified by the prescriber or based solely on the patient's self-report. Patients were identified only by their unique identifying number. ${ }^{5}$. Names and addresses were not included. We requested information on the following patients:

- Those on the program at September 30, 1989;

Those who entered the program in October,

November or December 1989; and,

Those who had an HIV test in October,

November or December 1989.

\section{RESULTS}

\section{Prescriber response}

Of the 13 prescribers who agreed to participate,

10 supplied the information. These 10 prescribers were treating around one-quarter of the patients in the program. Their patients were similar to other patients on the program in terms of sex, age, marital status and duration of narcotic use and dependence.

Most prescribers had to be followed up, some many times, before any forms were returned. The main reasons given for slow completion of forms were a lack of time (especially for those without secretarial support) and having to identify patients by number rather than by name.

\section{HIV testing practices}

The private prescribers in this study had incorporated routine HIV testing into their entry procedures and were about to begin or had already started regular retesting for patients in treatment. In the public clinic, procedures ranged from almost complete testing at the clinic to complete referral. Private patients, therefore, 


\section{HIV Testing in Methadone Patients}

\section{Continued from page 55}

were much more likely to have been tested and those who were tested during September-November 1989 were usually tested around the same time either by the prescriber or at a clinic with which that prescriber was associated.

\section{Data quality}

Data on HIV testing collected directly from prescribers may be more accurate, complete and/or reliable than that available on the treatment assessment form. Prescribers verified the HIV test results of most (91 per cent) of their patients. For the 70 per cent of patients whose most recent test was in September 1989 or later, this was usually because the prescribers had arranged the test.

As with the total population of methadone patients, HIV-related information for patients in this study was usually missing from their entry assessment forms (for example, HIV testing status had not been recorded for 64 per cent of the patients).

\section{HIV test results}

The prescribers indicated that 467 (35 per cent) of their patients had had an HIV test. Of these, 9 (2 per cent) were HIV-positive. All positive results were verified by the prescriber. This low HIV-positive rate is consistent with four recent studies of IVDU in western Sydney, in each of which fewer than 1 per cent of patients were found to be HIV-positive ${ }^{6,7}$.

Most patients (95 per cent) had been tested in 1989. Almost half (49 per cent) were tested between October and December 1989, usually by their methadone prescriber. Patients whose most recent HIV test was before October 1989 were similar to those who had not been tested in age, sex, marital status, employment status, partner's drug use, duration of drug use, duration of drug dependence and time spent in methadone programs. Those with children were more likely to have had an HIV test.

\section{CONCLUSIONS}

1 Prevalence and incidence data on HIV testing and status of patients in the Methadone Program can be obtained directly from methadone prescribers.

2 Considerable follow-up was needed to obtain voluntarily supplied data from this group of cooperative prescribers. If a system of compulsory supply of HIV testing data were to be introduced, the important issue of how to enforce this would need to be addressed. For example, prescribers tend to comply with the six-monthly prescription update because Departmental authority for them to continue to prescribe methadone is contingent upon it, whereas they leave many sections of the treatment assessment form unanswered because no such consequence applies.

The volume and quality of the data available from each prescriber will be related to the HIV testing practices of that prescriber. Those who do not directly arrange testing for their patients have difficulty supplying accurate, verified data, even of the most basic kind (date, place, result of
HIV test), since the confidentiality of HIV test results is protected by legislation. Accurate, updated information on individual prescribers' HIV testing practices is needed.

The Methadone Program's policy on HIV testing allows prescribers the full range from no testing or referrals whatsoever to the equivalent of comprehensive regular screening. The policy needs clarification.

5 Where prescribers have access to HIV testing information about their clients (for example, because they arrange regular HIV testing), the data supplied are likely to be more timely, accurate and complete than has been available from entry assessment forms.

6 The accuracy and comprehensiveness of data collected would be improved if prescribers were encouraged to arrange HIV testing, for example by making resources available to assist them with testing, counselling and/or data transcription.

HIV surveillance data obtained from prescribers can be successfully linked with patient characteristics on the Methadone Stats Unit database via the patients' unique identifying numbers.

8 Introduction of uniform and reliable HIV antibody screening procedures in the Methadone Program would assist efforts to gain a clearer picture of the prevalence and incidence of HIV in the patient population.

Judith E. Jones*, George Rubin*, Danny O'Connor**

* Epidemiology and Health Services Evaluation Branch

** Directorate of the Drug Offensive, NSW Health Department

The assistance of the Directorate of the Drug Offensive, in particular Stuart Riley of the NSW Methadone Program, and of the participating prescribers and clinics is gratefully acknowledged.

\section{EDITORIAL NOTE}

Following on from this study, a panel of NSW AIDS and drug researchers, clinicians and administrators was formed to draft strategies to improve the monitoring of HIV infection in IVDUs.

1. Forker, P, NSW Methadone Program Statistical Report for the Third Quarter. Sydney: Directorate of the Drug Offensive, NSW Department of Health, July 1989. By November, 1990 there were 5250 patients in the program.

2. Sandland, R, Estimation of the Number of Heroin Users in NSW using Police Arrest Data: Development of a Statistical Model. Sydney: NSW Drug and Alcohol Authority, April 1986.

3. Directorate of the Drug Offensive, Policies and Procedures for the Methadone Treatment of Opioid Dependence in NSW. Sydney: NSW Department of Health (undated).

4. The Methadone Stats Unit (Directorate of the Drug Offensive) supplied a de-identified database of patients in the methadone program (current to October 1989) which was derived from completed treatment admission forms.

5. The Department of Health assigns a unique identifying number to each patient who starts on the methadone program. Patients retain this number through different entries, exits and prescribers.

6. Bell, J, Fernandes, D \& Batey, R, Heroin users seeking methadone treatment. The Medical Journal of Australia, 152 (April 2), 1990.

7. Dwyer, $D$ et al, Low prevalence of human immunodeficiency virus infection in Methadone Program attenders and pregnant intravenous drug users in the western metropolitan region of Sydney. Australian. and New Zealand Journal of Medicine, 19, 1989. 